\title{
Low-Complexity Downlink User Selection for Massive MIMO Systems
}

\author{
Haijing Liu, Hui Gao, Member, IEEE, Shaoshi Yang, Member, IEEE, and Tiejun Lv, Senior Member, IEEE
}

\begin{abstract}
In this paper we propose a pair of low-complexity user selection schemes with zero-forcing precoding for multiuser massive MIMO downlink systems, in which the base station is equipped with a large-scale antenna array. First, we derive approximations of the ergodic sum rates of the systems invoking the conventional random user selection (RUS) and the locationdependant user selection (LUS). Then, the optimal number of simultaneously served user equipments (UEs), $K^{*}$, is investigated to maximize the sum rate approximations. Upon exploiting $K^{*}$, we develop two user selection schemes, namely $K^{*}$-RUS and $K^{*}$ LUS, where $K^{*}$ UEs are selected either randomly or based on their locations. Both of the proposed schemes are independent of the instantaneous channel state information of small-scale fading, therefore enjoying the same extremely-low computational complexity as that of the conventional RUS scheme. Moreover, both of our proposed schemes achieve significant sum rate improvement over the conventional RUS. In addition, it is worth noting that like the conventional RUS, the $K^{*}$-RUS achieves good fairness among UEs.
\end{abstract}

Index Terms-User selection, massive MIMO, low-complexity, system sum rate, user fairness.

\section{INTRODUCTION}

The multiuser MIMO (MU-MIMO) technology plays a key role in modern wireless communications due to its substantial performance gains over the conventional single-input single-output (SISO) techniques [1], [2]. Relying on MUMIMO, a multi-antenna base station (BS) can simultaneously serve multiple user equipments (UEs) within a cell using the same spectrum resource, and thus the spectral efficiency is improved. User selection is critical for optimizing MIMO systems' overall performance in a variety of scenarios and has been extensively studied, such as in cellular networks (see for example [3] and references therein) and in multi-hop networks [4]-[7]. The semi-orthogonal user selection (SUS) leveraging the degree of channel orthogonality among UEs is probably one of the most popular low-complexity user selection methods for improving system sum rates [3], [8], [9]. Additionally, considering the fairness amongst UEs, round robin scheduling [3] and random user selection (RUS) are regarded as the two simplest methods offering equal opportunities to all the

This work is financially supported by the National Natural Science Foundation of China (NSFC) (Grant No. 61271188, 61401041), the Fundamental Research Funds for the Central Universities (Grant No. 2014RC0106), and Beijing Municipal Science and Technology Commission research fund project No. D151100000115002.

Haijing Liu, Hui Gao, and Tiejun Lv are with the Beijing University of Posts and Telecommunications, Beijing, China 100876 (e-mail: \{Haijing_LIU, huigao, lvtiejun\} @bupt.edu.cn).

Shaoshi Yang is with the School of Electronics and Computer Science, University of Southampton, SO17 1BJ Southampton, U.K. (e-mail: sy7g09@ecs.soton.ac.uk).
candidate-UEs. Both of them have been widely employed in practical cellular systems as well [1].

In the realm of MU-MIMO, the recently proposed massive MIMO, where the BS is equipped with a large-scale antenna array to serve multiple UEs, has been widely envisaged as one of the major candidate technologies for the fifth generation (5G) cellular networks owing to its favorable features, such as huge spectral efficiency and energy efficiency gains [10][12], [14], [15]. Like in the conventional small-scale MUMIMO systems, user selection is also important in massive MU-MIMO systems [16]-[19], though it faces new challenges. More specifically, in the user selection for conventional MUMIMO systems, it is usually assumed that the number of candidate-UEs, $N$, is much larger than that of the BS antennas, $M$. Therefore, upon employing instantaneous channel state information (CSI)-aided user selection methods (e.g. SUS), multiuser diversity gains can be harvested to boost the overall system performance. By contrast, in massive MIMO systems, it is impractical to have $N \gg M$, since $M$ is already very large. Moreover, the computational complexity of the conventional user selection methods might be too high for the massive MIMO systems. For example, the computational complexity of SUS is roughly $\mathcal{O}\left(M^{3} N\right)$ [3], which will cause huge consumption of power and computational resources if $M$ becomes large.

Recently, a range of user selection schemes have been proposed for massive MIMO systems. The time-division duplex (TDD) and frequency-division duplex (FDD) based massive MIMO systems impose different requirements on user selection. By exploiting the instantaneous CSI of candidate-UEs, Lee et al. proposed an SUS-like user selection method in [18] and $\mathrm{Xu}$ et al. developed a greedy user selection scheme in [19]. These selection methods mainly focus on FDD scenarios, in which the amount of downlink transmission resources consumed by the downlink channel estimation training for all the candidate-UEs does not increase with the number of candidateUEs $N$ [20]. By contrast, in TDD scenarios, the downlink channel is estimated through uplink training relying on channel reciprocity, and the pilot/training symbol overhead imposed by channel estimation increases with $N$ [21]. In this scenario, if the number of candidate-UEs is large, most of the channel coherence slot in time domain will be consumed by channel estimation, leaving only a small fraction for downlink data transmission. Hence, besides the high computational complexity, the pilot overhead needed for channel estimation of candidateUEs also limits the application of instantaneous CSI-aided user selection methods in TDD scenarios. In addition, for FDD based massive MIMO systems, Nam et al. introduced 
user selection methods based on the candidate-UEs' feedback of instantaneous signal-to-interference-plus-noise ratio (SINR) in [17]. However, these methods are not applicable for TDD systems either. This is because in TDD scenarios, the feedback signals from a large number of candidate-UEs will increase the uplink proportion of the uplink-downlink shared frequency band at each coherence slot, resulting in reduced resources left for downlink data transmission. In summary, neither the instantaneous-CSI estimation based nor the uplink-feedback based user selection methods are suitable for the downlink of TDD-based massive MIMO systems. At the time of writing, the design of user selection for massive MIMO systems with the TDD mode remains a largely open area. Hence, the novel user selection methods which cause no or just little decrease of downlink transmission resources represent a new promising research subject.

In this paper, we consider the downlink of a TDD based massive MIMO system where pilot-based channel estimation and zero-forcing $(\mathrm{ZF})$ precoding are invoked for serving a number of UEs. First, with the aid of the random matrix theory (RMT)-based large system analysis, we derive approximations of the ergodic sum rates of the systems invoking the conventional RUS and the location-dependent user selection (LUS). The optimal number of simultaneously served UEs, denoted as $K^{*}$, is solved offline for maximizing the sum rate approximations. Then, aiming for improving the system sum rates, a pair of $K^{*}$-based low-complexity user selection methods are proposed, namely the $K^{*}$-based random user selection ( $K^{*}$-RUS) and the $K^{*}$-based location-dependant user selection ( $K^{*}$-LUS). For $K^{*}$-RUS, $K^{*}$ UEs are randomly selected for simultaneous data transmissions at each time slot. The system sum rates are improved with an appropriate configuration of $K^{*}$. Meanwhile, the fairness among UEs is guaranteed as a result of the random selection. For the $K^{*}$ LUS scheme, $K^{*}$ UEs nearest to the BS are selected for data transmission, which may achieve higher sum rate performance than $K^{*}$-RUS.

Notably, our schemes exhibit two fundamental differences as compared with the conventional user selection schemes. First, unlike the conventional SUS that requires the instantaneous CSI of small-scale fading (SSF), our schemes only need long-term CSI. Second, rather than emphasizing which UEs should be selected for improving system performance, the proposed user selection schemes mainly focus on how many UEs should be selected for simultaneous transmissions. Thanks to these differences, we bypass the complicated online computations regarding the sum rates and the selection metric, which are often inevitable in the conventional schemes. Therefore, the online computational complexity of the proposed two schemes is on the same order as that of the conventional RUS scheme. Furthermore, since our user selection schemes are independent of the SSF CSI of candidate-UEs, we no longer have to carry out channel estimation of all the candidate-UEs for user selection. Instead, only the active-UEs need to send pilots at each coherence slot. As a beneficial result, we are capable of saving the cost of channel training significantly and attaining more resources for data transmission.

It is worth pointing out that a location-adaptive transmission

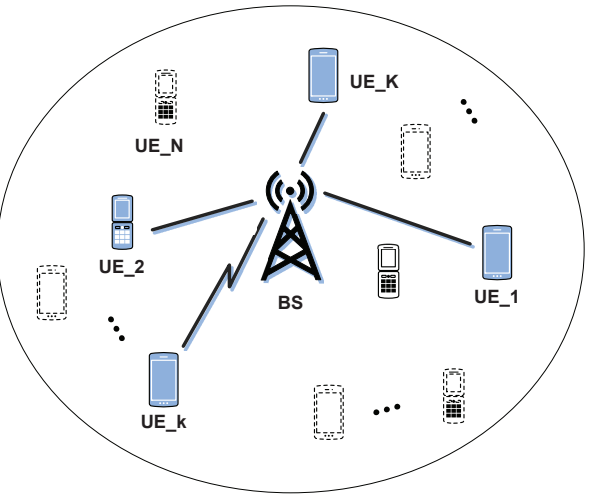

Fig. 1. The downlink of a TDD based massive MIMO system, which is composed of an $M$-antenna BS and $N$ single-antenna candidate-UEs. Among all the candidate-UEs, $K$ UEs are selected to be simultaneously served, which are regarded as the active-UEs.

strategy was proposed for TDD based massive MIMO systems in [22]. Our work differs from [22] in several respects. Random locations of UEs are assumed in this paper, whereas in [22] the UEs were assumed to be placed at fixed points. Therefore, the optimal number of active-UEs in our paper is independent of specific channel realizations, while in [22] this number has to be re-calculated whenever any candidate-UE's large-scale-fading (LSF) CSI changes. Moreover, we consider spatial correlation in the channel model, which is ignored in [22].

The remainder of this paper is organized as follows. The system model is presented in Section II. In Section III, we analyze the asymptotic sum rates of the system invoking the conventional RUS and LUS, and then further develop two lowcomplexity user selection schemes. Our numerical results are provided in Section IV. Finally, the conclusions are drawn in Section V.

Notations: We use uppercase and lowercase boldface letters to denote matrices and vectors, respectively. $(\cdot)^{H},(\cdot)^{\dagger}$ and $\operatorname{tr}(\cdot)$ denote the conjugate transpose, the pseudo-inverse, and the trace operations, respectively. $\mathrm{E}_{x}[\cdot]$ represents the expected value with respect to $x . \mathcal{C N}(\mathbf{m}, \mathbf{\Theta})$ denotes the circularly symmetric complex Gaussian distribution with mean vector $\mathbf{m}$ and covariance matrix $\Theta$. Finally, $\stackrel{\text { a.s. }}{\longrightarrow}$ denotes the almost sure convergence.

\section{SySTEM MODEL}

We consider the downlink of a TDD based massive MIMO system consisting of an $M$-antenna BS and $N$ single-antenna candidate-UEs $(N \geq M)$. We assume that $N$ and $M$ are of the same order. $K$ UEs $(K<M)$ are selected for simultaneous data transmissions at each coherence slot. The composite channel matrix $\mathbf{G} \in \mathbb{C}^{K \times M}$ from the BS to the $K$ active-UEs characterizes LSF, SSF and transmit correlation ${ }^{1}$, and can be expressed as

$$
\mathbf{G}=\mathbf{D}^{1 / 2} \mathbf{H R}^{1 / 2}
$$

${ }^{1}$ The distance between UEs is supposed to be sufficiently large compared to the signal wavelength, so the receive correlation is not taken into account. 
where $\mathbf{H} \in \mathbb{C}^{K \times M}$ is the $\mathrm{SSF}$ matrix with independent and identically distributed (i.i.d.) $\mathcal{C N}(0,1)$ entries, and the diagonal matrix $\mathbf{D} \in \mathbb{R}^{K \times K}$ contains the LSF coefficients $\beta_{k}$ along its main diagonal. We model the LSF of the $k$-th active$\mathrm{UE}$ as $^{2} \beta_{k}=c d_{k}^{-\alpha}, k=1, \ldots, K$, in which $d_{k}$ is the distance from the $k$-th active-UE to the BS, $\alpha$ is the pathloss exponent and $c$ is the pathloss at the reference distance. The transmit correlation matrix at the BS is modeled by the widely used $\delta$-Kac-Murdock-Szeg? matrix $\mathbf{R}$, in which $\delta$ is the antenna correlation coefficient [23].

The system operates in the TDD mode and the BS obtains the SSF CSI relying on the training-based channel estimation. The estimation $\hat{\mathbf{H}}$ of the SSF CSI matrix $\mathbf{H}$ is modeled as

$$
\mathbf{H}=\hat{\mathbf{H}}+\tilde{\mathbf{H}},
$$

where the $K \times M$ dimensional estimated channel matrix $\hat{\mathbf{H}}=$ $\left[\hat{\mathbf{h}}_{1}^{T}, \cdots, \hat{\mathbf{h}}_{K}^{T}\right]^{T}$ and the $K \times M$ dimensional error matrix $\tilde{\mathbf{H}}=$ $\left[\tilde{\mathbf{h}}_{1}^{T}, \cdots, \tilde{\mathbf{h}}_{K}^{T}\right]^{T}$ can be expressed as

$$
\hat{\mathbf{H}}=\sqrt{1-\rho} \mathbf{Z}_{1}, \quad \tilde{\mathbf{H}}=\sqrt{\rho} \mathbf{Z}_{2}, \quad 0 \leq \rho \leq 1 .
$$

$\hat{\mathbf{h}}_{k} \in \mathbb{C}^{1 \times M}$ and $\tilde{\mathbf{h}}_{k} \in \mathbb{C}^{1 \times M}$ are the $k$-th rows of $\hat{\mathbf{H}}$ and $\tilde{\mathbf{H}}$, respectively. Both $\mathbf{Z}_{1} \in \mathbb{C}^{K \times M}$ and $\mathbf{Z}_{2} \in \mathbb{C}^{K \times M}$ are composed of i.i.d. $\mathcal{C N}(0,1)$ entries, and the two matrices are independent with each other ${ }^{3}$. We assume that the LSF CSI of each UE and the transmit correlation matrix $\mathbf{R}$ are perfectly known at the BS. According to (2) and (3), the downlink channel matrix defined in (1) can be rewritten as ${ }^{4}$

$$
\begin{aligned}
\mathbf{G} & =\hat{\mathbf{G}}+\tilde{\mathbf{G}} \\
& =\mathbf{D}^{1 / 2} \hat{\mathbf{H}} \mathbf{R}^{1 / 2}+\mathbf{D}^{1 / 2} \tilde{\mathbf{H}} \mathbf{R}^{1 / 2} .
\end{aligned}
$$

Upon invoking the estimated channel matrix $\hat{\mathbf{G}}$ and the ZF precoding, the transmitted vector $\mathbf{x} \in \mathbb{C}^{M \times 1}$ is written as

$$
\begin{aligned}
\mathbf{x} & =\gamma \hat{\mathbf{G}}^{\dagger} \mathbf{s} \\
& =\gamma \hat{\mathbf{G}}^{H}\left(\hat{\mathbf{G}} \hat{\mathbf{G}}^{H}\right)^{-1} \mathbf{s},
\end{aligned}
$$

where $\gamma$ is the power controlling factor, $\mathbf{s}=\left[s_{1}, \cdots, s_{K}\right]^{T}$ is the $K \times 1$ information-bearing symbol vector and $s_{k}$ represents the symbol intended to the $k$-th active-UE. Denoting the available total transmit power at the $\mathrm{BS}$ as $P$, the long-term power constraint is given by

$$
\mathrm{E}\left[\operatorname{tr}\left(\mathbf{x} \mathbf{x}^{H}\right)\right] \leq P .
$$

Thus, $\gamma$ can be calculated as

$$
\gamma=\sqrt{\frac{P}{\operatorname{tr}\left(\hat{\mathbf{G}} \hat{\mathbf{G}}^{H}\right)^{-1}}} .
$$

\footnotetext{
${ }^{2}$ In this paper, we use a simplified LSF model in which the shadow fading is excluded. Nevertheless, it should be noted that algorithms and schemes developed in this paper can be directly extended to the model including shadow fading.

${ }^{3}$ Note that the imperfect CSI model invoked here is similar to that of [10] and different from that employed by [24] and [25]. In [24] and [25], the authors assumed $\hat{\mathbf{H}}=\mathbf{H}+\tilde{\mathbf{H}}$, where $\tilde{\mathbf{H}}$ and $\mathbf{H}$ are mutually independent.

${ }^{4}$ In fact, as shown in [26], the explicit forms of $\hat{\mathbf{G}}$ and $\tilde{\mathbf{G}}$ is related to the channel estimation approach, the power and the length of the training pilots, as well as the statistical information of the estimated channel. Here, we adopt a simplified model for tractability. Some tailored user selection schemes relying on a specific channel estimation algorithm (e.g., LS, MMSE) will be postponed for our future work.
}

It should be noted that we employ the equal power allocation among active-UEs for the sake of low computational complexity. Relying on (5), the $K \times 1$ dimensional received signal vector at the $K$ active-UEs is expressed as

$$
\begin{aligned}
\mathbf{y} & =\mathbf{G} \mathbf{x}+\mathbf{n} \\
& =\gamma \mathbf{s}+\gamma \tilde{\mathbf{G}} \hat{\mathbf{G}}^{\dagger} \mathbf{s}+\mathbf{n},
\end{aligned}
$$

where $\mathbf{n}=\left[n_{1}, \cdots, n_{k}\right]^{T} \in \mathbb{C}^{K \times 1}$ is the additive white Gaussian noise (AWGN) vector at the UEs, and $n_{k} \sim \mathcal{C N}\left(0, \sigma_{n}^{2}\right)$ represents the noise at the $k$-th active-UE. Additionally, the received signal received at the $k$-th active-UE is given by

$$
y_{k}=\gamma s_{k}+\gamma \sqrt{c d_{k}^{-\alpha}} \tilde{\mathbf{h}}_{k} \mathbf{R}^{1 / 2} \hat{\mathbf{G}}^{\dagger} \mathbf{s}+n_{k} .
$$

Then, we can write the SINR recorded at the $k$-th active-UE as

$$
\operatorname{SINR}_{k}=\frac{\gamma^{2}}{\sigma_{n}^{2}+c d_{k}^{-\alpha} \gamma^{2} \tilde{\mathbf{h}}_{k} \mathbf{R}^{1 / 2} \hat{\mathbf{G}}^{\dagger} \mathbf{s} \mathbf{s}^{H}\left(\hat{\mathbf{G}}^{\dagger}\right)^{H}\left(\mathbf{R}^{1 / 2}\right)^{H} \tilde{\mathbf{h}}_{k}^{H}} .
$$

Then, the sum rate $\mathcal{R}$ is given by

$$
\begin{aligned}
\mathcal{R} & =\left(1-\frac{K}{T}\right) \sum_{k=1}^{K} \mathcal{R}_{k} \\
& =\left(1-\frac{K}{T}\right) \sum_{k=1}^{K} \log _{2}\left(1+\operatorname{SINR}_{k}\right)
\end{aligned}
$$

where $\mathcal{R}_{k}$ is the rate of the $k$-th active-UE, and $T$ denotes the number of symbols over which the channel is constant. As a percentage, the pre-log factor $(1-K / T)$ implies that the downlink data transmission only occupies a fraction of the coherence slot. In particular, we assume that $K$ UEs are simultaneously served. Since each of the $K$ UEs is assigned one of the $K$ orthogonal pilot sequences, the length of the pilot sequence should not be shorter than $K$ symbols [11]. For simplicity, in this paper we adopt the shortest available pilot sequence of $K$-symbol length. Because the BS does not transmit data during the uplink pilot transmission for channel estimation, there exist $(T-K)$ symbols left for downlink data transmission at each coherence slot consisting of $T$ symbols. Therefore, the sum rate for downlink data transmission may be evaluated using (10). Note that in order to guarantee the feasibility of data transmission, we assume $K<T$ in this paper. Otherwise, the pilot transmission would occupy the entire coherence slot $T$.

\section{Asymptotic Sum Rate AnAlyses-BASED LOW-COMPLEXITY USER SELECTION}

In this section, the proposed user selection schemes are presented. First, relying on the RMT-based large system analysis, we derive a deterministic approximation of the ergodic sum rate of the $\mathrm{ZF}$ precoder aided massive MIMO system. This result brings new insights into the question of how to enhance the system sum rate performance. Then, a pair of low-complexity user selection schemes are proposed based on the attained approximation of the sum rate. 


\section{A. Sum Rate Approximation in the Large-System Regime}

We first evaluate the value of $\operatorname{SINR}_{k}$ for the scenario where $M$ and $K$ go to infinity with a finite ratio $M / K>0$. Then, the approximation of the ergodic sum rate is derived.

Applying RMT-based large-system analysis, we reveal that $\mathrm{SINR}_{k}$ may be characterized by (please see Appendix for the detailed derivation)

$$
\operatorname{SINR}_{k} \stackrel{a . s .}{\longrightarrow} \frac{1}{\sum_{i=1}^{K} d_{i}^{\alpha}\left(A(K, M)+B(K, M) d_{k}^{-\alpha}\right)},
$$

where

$$
\begin{aligned}
& A(K, M)=\frac{1}{1-\rho} \frac{\sigma_{n}^{2}}{\operatorname{Pc} \phi M}, \\
& B(K, M)=\frac{\rho}{1-\rho} \frac{\psi}{M \phi^{2}-K \psi} .
\end{aligned}
$$

In (12), $\phi$ is the unique solution of the equation

$$
\phi=\frac{1}{M} \operatorname{tr}\left(\mathbf{R}\left(\mathbf{I}_{M}+\frac{K}{M} \frac{1}{\phi} \mathbf{R}\right)^{-1}\right)
$$

and $\psi$ is defined as

$$
\psi=\frac{1}{M} \operatorname{tr}\left(\mathbf{R}^{2}\left(\mathbf{I}_{M}+\frac{K}{M} \frac{1}{\phi} \mathbf{R}\right)^{-2}\right) .
$$

Exploiting (10) and (11), the ergodic sum rate $\mathrm{E}[\mathcal{R}]$ in largesystem regime can be formulated as

$$
\begin{aligned}
\mathrm{E}[\mathcal{R}] & =\left(1-\frac{K}{T}\right) \mathrm{E}_{d_{1}, \cdots, d_{K}}\left[\sum_{k=1}^{K} \log _{2}\left(1+\mathrm{SINR}_{k}\right)\right] \\
& \geq \tilde{\mathcal{R}}
\end{aligned}
$$

in which $\tilde{\mathcal{R}}$ is defined as

$$
\tilde{\mathcal{R}}=\left(1-\frac{K}{T}\right) \sum_{k=1}^{K} \mathrm{E}_{d_{k}}\left[\log _{2}\left(1+\widetilde{\operatorname{SINR}}_{k}\right)\right]
$$

and " $\geq$ " is obtained by applying Jensen's inequality

$$
\mathrm{E}\left[\log _{2}\left(1+\frac{1}{x}\right)\right] \geq \log _{2}\left(1+\frac{1}{\mathrm{E}[x]}\right) .
$$

Additionally, $\widetilde{\operatorname{SINR}}_{k}$ is given by

$$
\widetilde{\operatorname{SINR}}_{k}=\frac{1}{T_{1} d_{k}^{\alpha}+T_{2} d_{k}^{-\alpha}+T_{3}},
$$

where

$$
\begin{aligned}
& T_{1}=A(K, M), \\
& T_{2}=B(K, M) \sum_{i=1, i \neq k}^{K} \mathrm{E}_{d_{i}}\left[d_{i}^{\alpha}\right], \\
& T_{3}=A(K, M) \sum_{i=1, i \neq k}^{K} \mathrm{E}_{d_{i}}\left[d_{i}^{\alpha}\right]+B(K, M) .
\end{aligned}
$$

In this paper, we employ the lower bound $\tilde{\mathcal{R}}$ defined by (13) as an approximation of the system sum rate. It should be emphasized that $\tilde{\mathcal{R}}$ is independent of the SSF CSI of UEs, which constitute the basis for our designs.
Remark 1. The results obtained above are invalid for the scenario of $K=M$ due to mathematical intractability. Fortunately, as shown by our simulation results that are given in Section IV-A, $K=M$ is rarely beneficial for enhancing the performance of the considered massive MIMO system. This phenomenon was also observed by [16] and [24]. Therefore, in this section we focus on the sum rate approximation for $K<M$.

\section{B. $K^{*}$-Based Random User Selection ( $\left.K^{*}-R U S\right)$}

In this subsection, we develop a novel RUS scheme, namely $K^{*}$-RUS, for the sake of improving the system sum rate and ensuring the fairness among candidate-UEs. Specifically, compared with the conventional RUS scheme in which $M$ UEs are selected for simultaneous data transmissions at each coherence slot, we modify the number of active-UEs to a more appropriate value $K^{*}$, which is decided according to the system parameters (e.g., the transmit power $P$ of the BS) and the statistical information of the channel (e.g., the probability distributions of SSF CSI and LSF CSI).

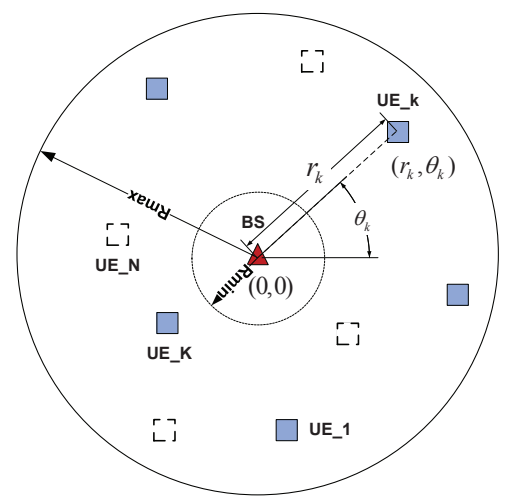

Fig. 2. The diagram of UE locations.

In order to obtain $K_{\mathrm{RUS}}^{*}$, we need to consider the user distribution, which facilitates characterizing the effects of LSF. As shown in Fig. 2, in this paper we employ the common circular cell model [27], where all the $N$ candidate-UEs are independently uniformly distributed (i.u.d.) in a circular cell having an inner radius of $R_{\min }$ and outer radius of $R_{\max }$, while the BS is located at the center point. We denote the locations in polar coordinates, i.e., $\left(r_{k}, \theta_{k}\right)$ is the location for the $k$-th candidate-UE and $(0,0)$ for the BS. Therefore, the probability density functions (PDFs) of $r_{k}$ and $\theta_{k}$ are given by (the subscript $k$ is omitted below for ease of notation)

$$
f_{R}(r)=\frac{2 r}{R_{\max }^{2}-R_{\min }^{2}}, \quad R_{\min } \leq r \leq R_{\max },
$$

and

$$
f_{\Theta}(\theta)=\frac{1}{2 \pi}
$$

respectively. The cumulative distribution functions (CDFs) of $r_{k}$ and $\theta_{k}$ are written as

$$
F_{R}(r)=\frac{r^{2}-R_{\min }^{2}}{R_{\max }^{2}-R_{\min }^{2}}, \quad R_{\min } \leq r \leq R_{\max },
$$


and

$$
F_{\Theta}(\theta)=\frac{\theta}{2 \pi}
$$

respectively.

As a result, for $K^{*}$-RUS, the PDF of the distance from the $k$-th active-UE to the BS, i.e. $f_{d_{k}}^{\mathrm{RUS}}(r)$, is given by

$$
f_{d_{k}}^{\mathrm{RUS}}(r)=f_{R}(r), \quad R_{\min } \leq r \leq R_{\max }, \quad k=1, \ldots, K .
$$

Then, it is easy to obtain

$$
\begin{aligned}
\sum_{i=1, i \neq k}^{K} \mathrm{E}_{d_{i}}\left[d_{i}^{\alpha}\right] & =(K-1) \int_{R_{\min }}^{R_{\max }} r^{\alpha} f_{R}(r) d r \\
& =\frac{2(K-1)\left(R_{\max }^{\alpha+2}-R_{\min }^{\alpha+2}\right)}{(\alpha+2)\left(R_{\max }^{2}-R_{\min }^{2}\right)} .
\end{aligned}
$$

Thus, $\tilde{\mathcal{R}}$ in (13) can be rewritten as

$$
\begin{aligned}
\tilde{\mathcal{R}}_{\mathrm{RUS}} & =\left(1-\frac{K}{T}\right) K \\
& \times \int_{R_{\min }}^{R_{\max }} \log _{2}\left(1+\frac{1}{T_{1} r^{\alpha}+T_{2} r^{-\alpha}+T_{3}}\right) f_{d_{k}}^{\mathrm{RUS}}(r) d r .
\end{aligned}
$$

Substituting (12) and (17) into (14), and then substituting (14) and (15) into (18), $\tilde{\mathcal{R}}_{\text {RUS }}$ can be reformulated as (19) which is given on the next page. We can see that (19) is only related to the system parameters of $T, M, K, R_{\min }, R_{\max }, P, c, \sigma_{n}^{2}, \rho, \alpha$ and $\delta$.

Given these system parameters, the optimal number $K_{\mathrm{RUS}}^{*}$ in the sense of $\tilde{\mathcal{R}}_{\text {RUS }}$ maximization can be obtained efficiently with an one-dimensional search over the candidate set $\{1,2, \ldots, M-1\}$, i.e.,

$$
\begin{aligned}
& K_{\mathrm{RUS}}^{*} \\
& \underset{K \in\{1, \ldots, M-1\}}{\arg \max } \Gamma_{1}\left(T, M, K, R_{\min }, R_{\max }, P, c, \sigma_{n}^{2}, \rho, \alpha, \delta\right) .
\end{aligned}
$$

Obviously, $K_{\mathrm{RUS}}^{*}$ is independent of any instantaneous CSI, which makes it possible to find $K_{\mathrm{RUS}}^{*}$ offline.

After obtaining $K_{\text {RUS }}^{*}$, the only online operation in the proposed $K^{*}$-RUS scheme is to randomly select $K_{\mathrm{RUS}}^{*}$ UEs for simultaneous data transmissions. Therefore, little extra computational complexity is imposed on the proposed $K^{*}$ RUS compared to the conventional RUS.

Remark 2. The authors of [24], [28] and [29] also discussed the optimal number of UEs for the ZF precoding, but they treated the LSF coefficients of UEs as deterministic values, which limit the generality of $K^{*}$. In other words, $K^{*}$ has to be updated whenever any LSF CSI of the system changes. By contrast, we take random UE locations into account for obtaining a more general and practical $K^{*}$. As long as the statistical properties of the system remain unchanged, our $K^{*}$ keeps its current value for arbitrary LSF and SSF channel realizations.

\section{C. $K^{*}$-Based Location-Dependant User Selection ( $\left.K^{*}-L U S\right)$}

In order to enhance the sum rate performance further, a $K^{*}$-based location-dependant user selection scheme, namely the $K^{*}$-LUS is developed in this subsection. In $K^{*}$-LUS, we select an appropriate number of UEs in descending order of the LSF coefficients (i.e., in ascending order of the BS-UE distances) for simultaneous data transmissions.

Inspired by $K^{*}$-RUS, let us first investigate the optimal number of active-UEs in LUS, which is denoted by $K_{\text {LUS }}^{*}$. We assume that $K$ UEs are selected and the distances between the selected UEs and the BS satisfy $d_{1} \leq d_{2} \leq \cdots \leq d_{K}$. Let $\left(r_{k}, \theta_{k}\right)$ represent the $k$-th active-UE according to the distance in ascending order. According to (16), we can get the PDF of the order statistic $d_{k}, k=1, \ldots, K$ in LUS as

$$
\begin{aligned}
f_{d_{k}}^{\mathrm{LUS}}\left(r_{k}\right) & =\frac{1}{\mathrm{~B}(k, N-k+1)} F_{R}^{(k-1)}\left(r_{k}\right)\left[1-F_{R}\left(r_{k}\right)\right]^{N-k} f_{R}\left(r_{k}\right) \\
& =\frac{2 r_{k}\left(R_{\max }^{2}-r_{k}^{2}\right)^{N-k}\left(r_{k}^{2}-R_{\min }^{2}\right)^{k-1}}{\mathrm{~B}(k, N-k+1)\left(R_{\max }^{2}-R_{\min }^{2}\right)^{N}},
\end{aligned}
$$

in which $\mathrm{B}(x, y)$ represents the Beta function with parameters $x$ and $y$. Then, we have

$$
\mathrm{E}_{d_{k}}\left[d_{k}^{\alpha}\right]=R_{\min 2}^{\alpha} F_{1}\left(k,-\frac{\alpha}{2} ; N+1 ; 1-\frac{R_{\max }^{2}}{R_{\min }^{2}}\right)
$$

and

$$
\begin{aligned}
& \mathrm{E}_{d_{k}}\left[\log _{2}\left(1+{\widetilde{\operatorname{SINR}_{k}}}_{k}\right)\right] \\
& =\int_{R_{\min }}^{R_{\max }} \log _{2}\left(1+\frac{1}{T_{1} r_{k}^{\alpha}+T_{2} r_{k}^{-\alpha}+T_{3}}\right) f_{d_{k}}^{\mathrm{LUS}}\left(r_{k}\right) d r_{k}
\end{aligned}
$$

where ${ }_{2} F_{1}(\cdot)$ is the ordinary hypergeometric function [30]. Therefore, $\tilde{\mathcal{R}}_{\text {LUS }}$ can be formulated with the aid of (12), (14), (21), (22) and (23), as shown in (24) on the next page.

If the system parameters are fixed, we are capable of solving for the optimal number $K_{\mathrm{LUS}}^{*}$ maximizing $\tilde{\mathcal{R}}_{\mathrm{LUS}}$ offline relying on standard line search algorithms, i.e.,

$$
\begin{aligned}
& K_{\mathrm{LUS}}^{*} \\
& \underset{K \in\{1, \ldots, M-1\}}{\arg \max } \Gamma_{2}\left(T, M, K, R_{\min }, R_{\max }, P, c, \sigma_{n}^{2}, \rho, \alpha, \delta\right) .
\end{aligned}
$$

As long as we find $K_{\mathrm{LUS}}^{*}$, we just need to sort UEs in ascending order of their distances from the BS and select the first $K_{\mathrm{LUS}}^{*}$ UEs for data transmission.

Remark 3. With respect to the number of active-UEs $K$, we determine $K=K_{\mathrm{RUS}}^{*}$ and $K=K_{\mathrm{LUS}}^{*}$ according to the given system parameters in the proposed $K^{*}$-RUS and $K^{*}$ LUS schemes, respectively. By contrast, there usually exists the scenario of $K=M$, where the full-spatial-multiplexing transmission may be performed in the conventional RUS and LUS schemes. In SUS, the value of $K^{*}$ depends on specific channel realizations and will not be obtained until the selection procedure is completed (please see Section IV-B and Section VI-A in [3] for more details). 


$$
\begin{aligned}
& \tilde{\mathcal{R}}_{\mathrm{RUS}} \\
& =\Gamma_{1}\left(T, M, K, R_{\min }, R_{\max }, P, c, \sigma_{n}^{2}, \rho, \alpha, \delta\right) \\
& =\left(1-\frac{K}{T}\right) K \\
& \times \int_{R_{\min }}^{R_{\max }} \frac{2 r}{R_{\max }^{2}-R_{\min }^{2}} \log _{2}\left(1+\frac{1-\rho}{\frac{\sigma_{n}^{2}}{P c \phi M}\left(r^{\alpha}+\frac{2(K-1)\left(R_{\max }^{\alpha+2}-R_{\min }^{\alpha+2}\right)}{(\alpha+2)\left(R_{\max }^{2}-R_{\min }^{2}\right)}\right)+\frac{\rho \psi}{M \phi^{2}-K \psi}\left(\frac{2(K-1)\left(R_{\max }^{\alpha+2}-R_{\min }^{\alpha+2}\right)}{(\alpha+2)\left(R_{\max }^{2}-R_{\min }^{2}\right)} r^{-\alpha}+1\right)}\right) d r .
\end{aligned}
$$

$$
\begin{aligned}
& \tilde{\mathcal{R}}_{\mathrm{LUS}} \\
& =\Gamma_{2}\left(T, M, K, R_{\min }, R_{\max }, P, c, \sigma_{n}^{2}, \rho, \alpha, \delta\right) \\
& =\left(1-\frac{K}{T}\right) \sum_{k=1}^{K} \int_{R_{\min }}^{R_{\max }} \frac{2 r_{k}\left(R_{\max }^{2}-r_{k}^{2}\right)^{N-k}\left(r_{k}^{2}-R_{\min }^{2}\right)^{k-1}}{\mathrm{~B}(k, N-k+1)\left(R_{\max }^{2}-R_{\min }^{2}\right)^{N}} \log _{2}(1+ \\
& \left.\frac{1-\rho}{\frac{\sigma_{n}^{2}}{P c \phi M}\left(r_{k}^{\alpha}+R_{\min 2}^{\alpha} F_{1}\left(k,-\frac{\alpha}{2} ; N+1 ; 1-\frac{R_{\max }^{2}}{R_{\min }^{2}}\right)\right)+\frac{\rho \psi}{M \phi^{2}-K \psi}\left(r_{k}^{-\alpha} R_{\min 2}^{\alpha} F_{1}\left(k,-\frac{\alpha}{2} ; N+1 ; 1-\frac{R_{\max }^{2}}{R_{\min }^{2}}\right)+1\right)}\right) d r_{k} .
\end{aligned}
$$

\section{Computational Complexity Analysis}

For a system having $M$ BS antennas and $N$ candidateUEs, although the user selection relying on exhaustive search achieves the best sum rate performance, approximately $\sum_{k=1}^{M}\left(\begin{array}{c}N \\ k\end{array}\right) k^{5} M$ complex-valued operations are required to complete one selection [31], which may be unaffordable in practice. For SUS, the computational complexity is roughly $\mathcal{O}\left(M^{3} N\right)$ [3], which is high for large- $M$ systems. In stark contrast to these conventional schemes, the online computational complexity of the proposed $K^{*}$-RUS and $K^{*}$-LUS is independent of $M$ and $N$, and the instantaneous CSI-based complicated online computations are avoided. As a result, the computational complexity is approximately $\mathcal{O}(1)$, which is just the same as that of the conventional RUS scheme.

\section{E. Performance Analysis for the Special Case of $\rho=0, \delta=0$}

All the above investigations are subject to the general case, i.e., in the context of the systems with imperfect CSI and transmit antenna correlation. In this subsection, we consider a special case in which there exists neither channel estimation error nor transmit antenna correlation, i.e., $\rho=0, \delta=0$. In this context, because we can obtain clearer insights into how the system performance is affected by different user selection schemes.

For $\rho=0, \delta=0$, we have

$$
\begin{aligned}
& A(K, M)=\frac{\sigma_{n}^{2}}{\operatorname{Pc}(M-K)}, \\
& B(K, M)=0 .
\end{aligned}
$$

Substituting them into (19) and (24), the approximate sum rates can be calculated. Nevertheless, the integrals of logarithmic functions in (19) and (24) degrade the intelligibility of the results.
Here, a new method, which is different from those adopted in Section III-B and Section III-C, is developed for finding a much simpler expression of the ergodic sum rate approximation in this special case. In what follows we apply Jensen's inequality in a slightly different manner for the sake of finding a more concise expression of the system sum rate approximation. More specifically, we have

$$
\begin{aligned}
\mathrm{E}[\mathcal{R}] & =\left(1-\frac{K}{T}\right) \mathrm{E}_{d_{1}, \ldots, d_{K}}\left[\sum_{k=1}^{K} \log _{2}\left(1+\operatorname{SINR}_{k}\right)\right] \\
& \geq\left(1-\frac{K}{T}\right) K \log _{2}(1+\widetilde{\widehat{\operatorname{SINR}}})
\end{aligned}
$$

where

$$
\widetilde{\widetilde{\mathrm{SINR}}}=\frac{P c(M-K)}{\sigma_{n}^{2} \sum_{k=1}^{K} \mathrm{E}_{d_{k}}\left[d_{k}^{\alpha}\right]} .
$$

The approximate sum rate $\tilde{\tilde{\mathcal{R}}}$ for $K^{*}$-RUS is then given by

$$
\begin{aligned}
& \tilde{\tilde{\mathcal{R}}}_{\mathrm{RUS}}^{*}=\left(1-\frac{K_{\mathrm{RUS}}^{*}}{T}\right) K_{\mathrm{RUS}}^{*} \\
& \times \log _{2}\left(1+\frac{P c\left(M-K_{\mathrm{RUS}}^{*}\right)(\alpha+2)\left(R_{\mathrm{max}}^{2}-R_{\mathrm{min}}^{2}\right)}{2 \sigma_{n}^{2} K\left(R_{\mathrm{max}}^{\alpha+2}-R_{\mathrm{min}}^{\alpha+2}\right)}\right) .
\end{aligned}
$$

For $K^{*}$-LUS, the sum rate is approximated as

$$
\begin{aligned}
& \tilde{\tilde{\mathcal{R}}}_{\mathrm{LUS}}^{*}=\left(1-\frac{K_{\mathrm{LUS}}^{*}}{T}\right) K_{\mathrm{LUS}}^{*} \\
& \times \log _{2}\left(1+\frac{P c\left(M-K_{\mathrm{LUS}}^{*}\right)}{\sigma_{n}^{2} \sum_{k=1}^{K_{\mathrm{LUS}}^{*}} R_{\mathrm{min} 2}^{\alpha} F_{1}\left(k,-\frac{\alpha}{2} ; N+1 ; 1-\frac{R_{\max }^{2}}{R_{\min }^{2}}\right)}\right) .
\end{aligned}
$$

Compared to (19) and (24), there is no integral calculation of logarithmic functions in (26) and (27), which simplifies the 
system sum rate expression. Furthermore, as shown in Fig. 3, (26) and (27) are capable of providing tight approximations in the case of $\rho=0, \delta=0$.

According to (26) and (27), it is clear that both of the sum rates $\tilde{\tilde{\mathcal{R}}}_{\mathrm{RUS}}^{*}$ and $\tilde{\tilde{\mathcal{R}}}_{\text {LUS }}^{*}$ increase when $P$ and $M$ become larger. Additionally, when $N$ increases, the sum rate of $K^{*}$-LUS increases, while the sum rate of $K^{*}$-RUS remains unchanged.

As far as SUS is concerned in the TDD scenario, as proved in [3], the ergodic sum rate is upper bounded by $\overline{\mathcal{R}}_{\text {SUS }}$, which is given by

$$
\overline{\mathcal{R}}_{\text {SUS }}=\left[1-\frac{N}{T}\right]^{+} M \Theta\left(\log _{2} \log _{2} N\right),
$$

where $[\cdot]^{+}$is defined as $[x]^{+}=\max \{x, 0\}$. Due to the pilot overhead imposed by the channel estimation, the system sum rate is scaled by the factor $[1-N / T]^{+}$. More specifically, in this case the instantaneous CSI of all the $N$ candidate-UEs are required for select ingactive-UEs at each coherence slot.

Comparing the pre-log parts $[1-N / T]^{+}$in (28), (1$\left.K_{\mathrm{RUS}}^{*} / T\right)$ in (26) and $\left(1-K_{\mathrm{LUS}}^{*} / T\right)$ in (27), we can see that the advantages of $K^{*}$-RUS and $K^{*}$-LUS are obvious for the systems relying on pilot-based channel estimation. In particular, when $T$ is not significantly larger than $N$, with regard to SUS, a large portion of the coherence slot would be dedicated to channel estimation, which reduces the resources for the downlink data delivery. By contrast, with the proposed user selection schemes, we only have to estimate the CSI of the $K_{\mathrm{RUS}}^{*}$ or $K_{\mathrm{LUS}}^{*}$ active-UEs for precoding. Both $K_{\mathrm{RUS}}^{*}$ and $K_{\text {LUS }}^{*}$ are usually much smaller than $N$, hence our schemes are superior to SUS by exploiting more data transmission resources.

Remark 4. The result of $\Theta\left(\log _{2} \log _{2} N\right)$ in (28) is obtained when we have $N \rightarrow \infty$ and a fixed $M$ [3]. Moreover, as shown in [32], when the number of candidate-UEs $N$ is linearly related to the number of $\mathrm{BS}$ antennas $M$ (i.e. the case we have discussed in this paper), only marginal multiuser diversity gain might be achieved by SUS.

\section{Numerical Simulations AND Discussions}

In this section we present simulation results to show the benefits of the proposed user selection schemes. The cellular model employed is based on that of [16] and [33]. Like [10], we assume the number of symbols in a coherence slot is 196 , over which the channel is constant, i.e., $T=196$. The cell radius is $R_{\max }=250 \mathrm{~m}$ and the minimum distance is $R_{\min }=$ $35 \mathrm{~m}$. The pathloss exponent is $\alpha=3.76$ and the reference LSF factor is $c=10^{-3.53}$. The total noise power is assumed as $\sigma_{\mathrm{n}}^{2}=-96 \mathrm{dBm}$.

\section{A. Sum Rate Performance}

In Fig. 3, the sum rate performance of $K$-RUS and $K$ LUS as a function of $K$ is evaluated in the case of $P=30$ $\mathrm{dBm}, M=32$ and $N=64$. The simulated ergodic sum rates (marked as 'Sim.' in the figure) are obtained by averaging over 10000 independent channel realizations (both SSF and LSF CSI are regenerated at each realization). It is observed that

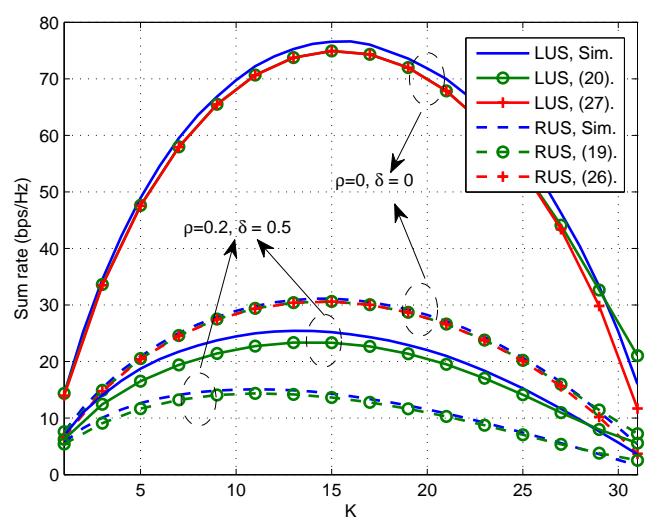

Fig. 3. Sum rates of $K$-RUS and $K$-LUS. $P=30 \mathrm{dBm}, M=32, N=64$.
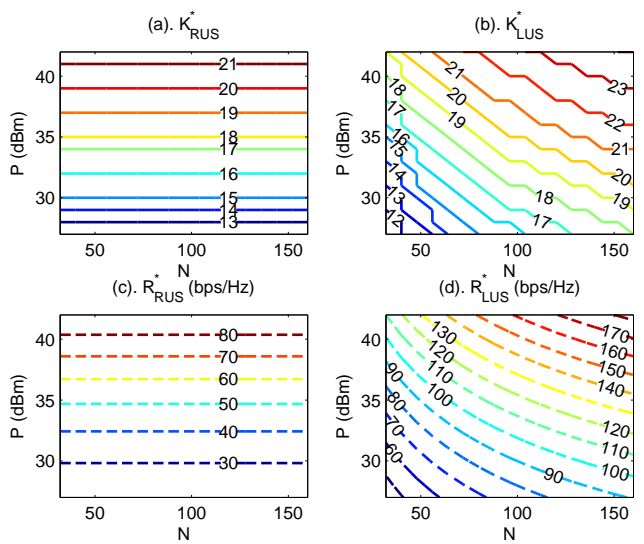

Fig. 4. Contour figures of $K_{\mathrm{RUS}}^{*}, K_{\mathrm{LUS}}^{*}, R_{\mathrm{RUS}}^{*}$ and $R_{\mathrm{LUS}}^{*}$ against $P$ and $N$. $\rho=0, \delta=0, M=32$. $K_{\mathrm{RUS}}^{*}$ and $K_{\mathrm{LUS}}^{*}$ are shown in (a) (i.e., the top-left sub figure) and (b) (i.e., the top-right sub figure) with solid lines, respectively. $R_{\mathrm{RUS}}^{*}$ and $R_{\mathrm{LUS}}^{*}$ are shown in (c) (i.e., the bottom-left sub figure) and (d) (i.e., the bottom-right sub figure) with dash lines, respectively.

there exist $K_{\mathrm{RUS}}^{*}$ and $K_{\mathrm{LUS}}^{*}$ which maximize the system sum rate for $K$-RUS and $K$-LUS, respectively. Moreover, we have $K_{\mathrm{RUS}}^{*}<M$ and $K_{\mathrm{LUS}}^{*}<M$ for the simulation parameters considered. In Fig. 3 we also show the approximate sum rates given by (19) and (24), which are very tight. Therefore, it is reasonable to design user selection schemes based on them.

In order to provide intuitive insights into how $K_{\mathrm{RUS}}^{*}$ and $K_{\text {LUS }}^{*}$ are affected by $P$ and $N$, we evaluated $K_{\text {RUS }}^{*}$ and $K_{\text {LUS }}^{*}$ with no transmit correlation and perfect CSI estimation (i.e., $\rho=0, \delta=0$ ) in Fig. 4 (a) and (b), respectively. It is observed that both $K_{\mathrm{RUS}}^{*}$ and $K_{\mathrm{LUS}}^{*}$ increase with the transmit power $P$. On the other hand, when we increase $N, K_{\text {LUS }}^{*}$ increases and $K_{\text {RUS }}^{*}$ remains unchanged. This can be easily explained from the perspective of multiuser diversity gain. In particular, $K_{\text {LUS }}^{*}$ is related to $N$ because we select UEs according to LSF in $K^{*}$-LUS. By contrast, $K_{\mathrm{RUS}}^{*}$ is independent of $N$ because random selection is adopted in $K^{*}$-RUS. Moreover, Fig. 4 (c) and Fig. 4 (d) provide the sum rates of $K^{*}$-RUS and $K^{*}$ LUS. As expected, we can see that both $\mathcal{R}_{\text {RUS }}^{*}$ and $\mathcal{R}_{\text {LUS }}^{*}$ are enhanced by the increase of $P$. When $N$ increases, $\mathcal{R}_{\text {LUS }}^{*}$ rises but $\mathcal{R}_{\mathrm{RUS}}^{*}$ remains unchanged.

In Fig. 5 the impacts of channel estimation accuracy $\rho$ and 

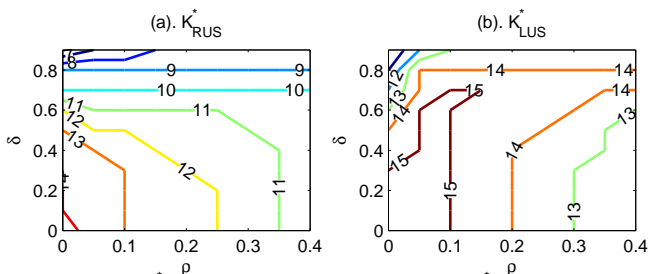

(c). $R_{\text {RUS }}^{*}(b p s / H z)$
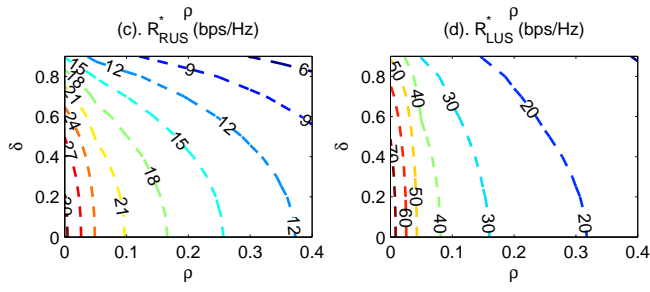

Fig. 5. Contour figures of $K_{\mathrm{RUS}}^{*}, K_{\mathrm{LUS}}^{*}, R_{\mathrm{RUS}}^{*}$ and $R_{\mathrm{LUS}}^{*}$ against $\rho$ and $\delta$. $P=30 \mathrm{dBm}, M=32, N=64 . K_{\mathrm{RUS}}^{*}$ and $K_{\mathrm{LUS}}^{*}$ are shown in (a) and (b) with solid lines, respectively. $R_{\mathrm{RUS}}^{*}$ and $R_{\mathrm{LUS}}^{*}$ are shown in (c) and (d) with dash lines, respectively.

channel correlation factor $\delta$ on the system performance are characterized, where we set $P=30 \mathrm{dBm}, M=32$ and $N=$ 64 . In sub-figures (a) and (b), the optimal number of activeUEs for $K^{*}$-RUS and $K^{*}$-LUS are shown, respectively. We can see that both $K_{\mathrm{RUS}}^{*}$ and $K_{\mathrm{LUS}}^{*}$ attain their maximum values at $\rho=0, \delta=0$. This observation indicates that the BS should serve more UEs under uncorrelated channel scenarios with perfect CSI estimation than those under correlated channel scenarios with imperfect CSI estimation. The sum rates of $K^{*}$-RUS and $K^{*}$-LUS are shown in the sub-figures (c) and (d), respectively. It is clear that the sum rates decrease as $\delta$ and $\rho$ increase.

The sum rate performance of various user selection schemes, including $K^{*}$-LUS, $K^{*}$-RUS, SUS and RUS, is evaluated against the transmit power $P$ with $M=32, N=64$ in Fig. 6. Four scenarios are investigated, i.e., uncorrelated channel with perfect CSI estimation $(\rho=0, \delta=0)$, correlated channel with perfect CSI estimation ( $\rho=0, \delta=0.5)$, uncorrelated channel with imperfect CSI estimation $(\rho=0.1, \delta=0)$ and correlated channel with imperfect CSI estimation $(\rho=$ $0.1, \delta=0.5)$. Note that for the conventional RUS, we randomly select $M$ UEs for simultaneous data transmissions. For SUS, in order to ensure fair comparisons among all the four schemes, we adopt the equal power allocation instead of the water filling allocation. Furthermore, the optimal value of $\alpha_{\text {SUS }}$, which is an important parameter in SUS (described as $\alpha$ in [3]), is used for the SUS scheme in our simulations ${ }^{5}$. As expected, $K^{*}$-LUS achieves the best sum rate performance among the four schemes, and compared with RUS, $K^{*}$-RUS also achieves a significant sum rate improvement. In addition, due to the non-negligible channel estimation pilot overhead and the lack of multiuser diversity gain, the conventional SUS scheme achieves similar [e.g., in sub-figures (a) and (b)] or even worse [e.g., in sub-figures (c) and (d)] sum rate

\footnotetext{
${ }^{5}$ The sum rate performance of the SUS scheme is highly sensitive to the choice of $\alpha_{\text {SUS }}$. The optimal value of $\alpha_{\text {SUS }}$ varies with the changes of $M, N$ and $P$. By means of searching over the interval $(0,1]$, we obtain optimal values of $\alpha_{\text {SUS }}$ maximizing the sum rates of the SUS scheme for different configurations of $M, N$ and $P$.
}
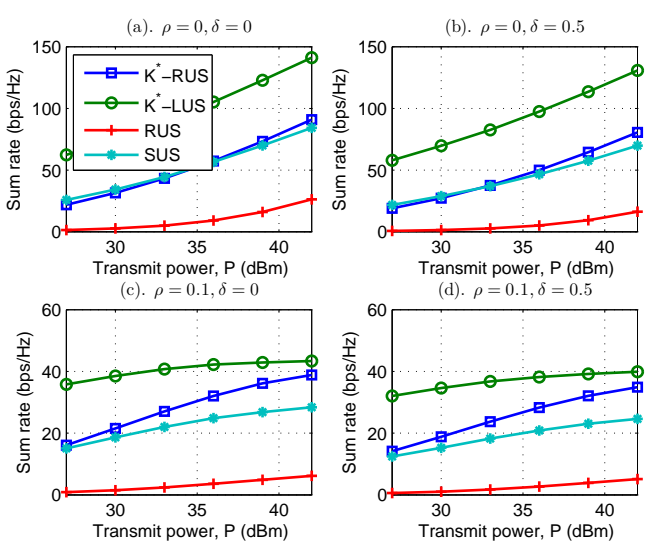

Fig. 6. Sum rate $\mathcal{R}$ vs. transmit power P. $M=32$ and $N=64$.
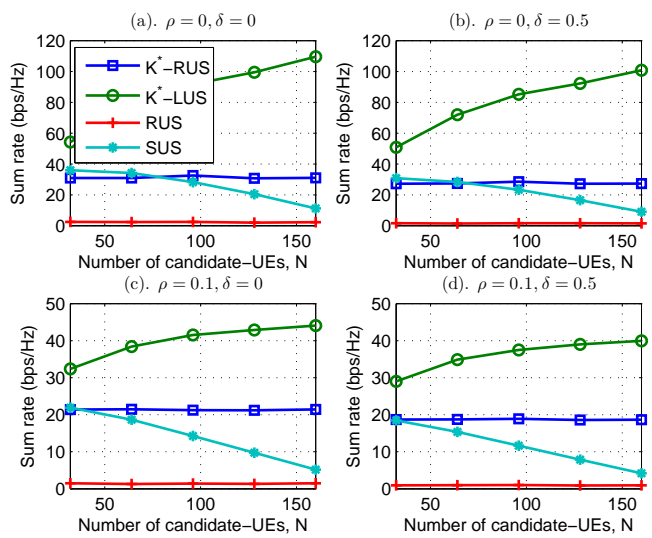

Fig. 7. Sum rate $\mathcal{R}$ vs. the number of candidate-UEs $N . P=$ $30 \mathrm{dBm}$ and $M=32$.

performance than the proposed $K^{*}$-RUS, even though the online computational complexity of SUS is much higher than that of $K^{*}$-RUS.

Furthermore, in Fig. 7, we investigate the sum rates of the four schemes for different numbers of candidate-UEs, $N$, when $P=30 \mathrm{dBm}$ and $M=32$. It is clear that with the increase of $N$, the sum rates of $K^{*}$-RUS and RUS remain unchanged. In contrast, $K^{*}$-LUS obtains sum rate improvements as $N$ increases because more multiuser diversity related to LSF can be exploited with larger $N$. For SUS, the sum rate decreases with $N$, because the pilot overhead becomes serious for large $N$, which overwhelms the increase of the multiuser diversity gain.

\section{B. Fairness Performance}

In this subsection, we evaluate the system performance in terms of long-term fairness among UEs. The Jain's Fairness Index (JFI) [34] $\mathcal{F}$, defined as

$$
\mathcal{F}=\frac{\left(\sum_{n=1}^{N} \omega_{n} \mathcal{R}_{n}\right)^{2}}{N \sum_{n=1}^{N}\left(\omega_{n} \mathcal{R}_{n}\right)^{2}},
$$

is employed in our investigation, where $\mathcal{R}_{n}$ is the rate of the $n$-th candidate-UE defined in (10) and $\omega_{n}$ is the probability 

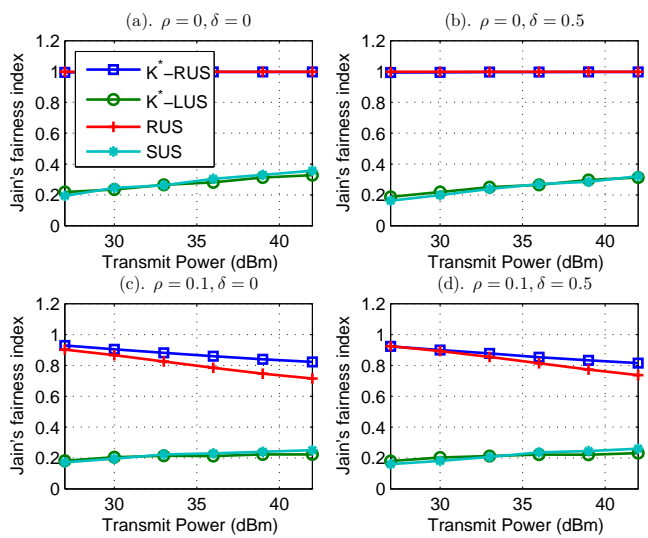

Fig. 8. Fairness performance $\mathcal{F}$ vs. transmit power $P . M=32$ and $N=64$.

of the $n$-th candidate-UE being selected to be served at each coherence slot.

First, we briefly analyze the long-term fairness performance of $K^{*}$-RUS and $K^{*}$-LUS without considering the channel estimation error and transmit correlation. Then the simulation results are shown in Fig. 8 and Fig. 9.

In the case of $\rho=0, \delta=0$, for the $n$-th candidate-UE in $K^{*}$-RUS, we have $\omega_{n}=K_{\mathrm{RUS}}^{*} / N$ and $\mathcal{R}_{n}=\mathcal{R}_{\mathrm{RUS}}^{*} / K_{\mathrm{RUS}}^{*}$. Therefore, the JFI of $K^{*}$-RUS is given by

$$
\mathcal{F}_{\mathrm{RUS}}=\frac{\left(\sum_{n=1}^{N} \frac{K_{\mathrm{RUS}}^{*}}{N} \frac{\mathcal{R}_{\mathrm{RUS}}^{*}}{K_{\mathrm{RUS}}^{*}}\right)^{2}}{N \sum_{n=1}^{N}\left(\frac{K_{\mathrm{RUS}}^{*}}{N} \frac{\mathcal{R}_{\mathrm{RUS}}^{*}}{K_{\mathrm{RUS}}^{*}}\right)^{2}}=1 .
$$

It is clear that $K^{*}$-RUS is capable of offering the optimal fairness among candidate-UEs. For $K^{*}$-LUS, the $K_{\text {LUS }}^{*}$ candidateUEs near to the BS are always active, whereas the other UEs far from the BS have little chance to be served. Thus, for the candidate-UE which is the $i$-th nearest to the BS, we have $\omega_{i}=1, \mathcal{R}_{i}=\mathcal{R}_{\mathrm{LUS}}^{*} / K_{\mathrm{LUS}}^{*}, 1 \leq i \leq K_{\mathrm{LUS}}^{*}$, and $\omega_{i}=0, \mathcal{R}_{i}=0, K_{\mathrm{LUS}}^{*}<i \leq N$. The JFI of $K^{*}$-LUS is then calculated as

$$
\mathcal{F}_{\mathrm{LUS}}=\frac{\left(\sum_{n=1}^{K_{\mathrm{LUS}}^{*}} \frac{\mathcal{R}_{\mathrm{LUS}}^{*}}{K_{\mathrm{LUS}}^{*}}\right)^{2}}{N \sum_{n=1}^{K_{\mathrm{LUS}}^{*}}\left(\frac{\mathcal{R}_{\mathrm{LUS}}^{*}}{K_{\mathrm{LUS}}^{*}}\right)^{2}}=\frac{K_{\mathrm{LUS}}^{*}}{N} .
$$

Usually, we have $\mathcal{F}_{\text {LUS }}<1$ because of $K_{\text {LUS }}^{*}<N$. Moreover, we can see that $\mathcal{F}_{\text {LUS }}$ increases when $K_{\text {LUS }}^{*}$ becomes higher.

In Fig. 8 we show the fairness among UEs against $P$ in the context of various schemes with $M=32, N=64$. In this simulation, we assume that the LSF CSI of each candidateUE remains unchanged for $100 T$ and the SSF CSI changes for each $T$. The window length evaluated for JFI is also assumed to be $100 T$ for creating the worst scenario in terms of fairness. We can see that $K^{*}$-RUS provides good fairness among UEs, and $K^{*}$-LUS exhibits poor fairness performance. With perfect channel estimation, the JFI of $K^{*}$-RUS maintains 1 for various $P$, which is consistent with (29). Furthermore, for $K^{*}$-LUS, the JFI increases as $P$ increases. This is because with the increase of $P$, more UEs can be served at the same time while $N$ keeps unchanged, which coincides with (30).
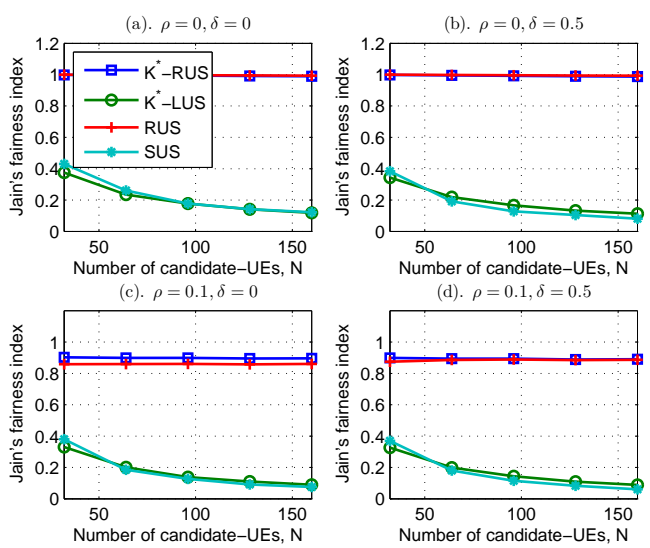

Fig. 9. Fairness performance $\mathcal{F}$ vs. the number of candidate-UEs $N . P=$ $30 \mathrm{dBm}$ and $M=32$.

Note that we also give the simulation results concerning the fairness performance with imperfect channel estimation. In this context, the JFI of $K^{*}$-RUS decreases as $P$ increases. This is because the SINRs of active-UEs are different from each other due to the existence of the second part of the denominator of (9) (i.e., the part related to the channel estimation error). Moreover, the SINR difference increases when $P$ rises. As a result, the fairness performance decreases.

Finally, the fairness performance against $N$ for $M=$ $32, P=30 \mathrm{dBm}$ is shown in Fig. 9. It can be observed that $K^{*}$-RUS is capable of achieving good fairness performance, whereas $K^{*}$-LUS has poor fairness performance. Moreover, the increasing of $N$ degrades the fairness performance of $K^{*}$ LUS, because the proportion of active-UEs in candidate-UEs declines although $K_{\text {LUS }}^{*}$ increases as $N$ becomes larger.

\section{CONCLUSIONS}

Considering the requirements of high energy efficiency and massive device connectivity in the future $5 \mathrm{G}$ communication systems, we have proposed a pair of low-complexity user selection methods for downlink massive MIMO systems in this paper. Taking the randomness of both the channel matrix and UE locations into consideration, we have obtained the approximations of the ergodic sum rates for the multiuser massive MIMO systems. By exploiting these approximations, $K^{*}$-RUS and $K^{*}$-LUS are developed, which are capable of significantly enhancing the system sum rate performance. Since no online operations related to SSF CSI are required in the proposed user selection algorithms, the computational complexity of the proposed schemes is extremely low. Besides the sum rate improvements, we also investigated the fairness among UEs and showed the remarkable fairness performance advantages of the proposed $K^{*}$-RUS scheme. In the future, we will investigate low-complexity user selection methods in multi-cell scenarios. 


\section{DETAILED DERIVATION OF (11)}

With (3) and (6), we have

$$
\gamma^{2}=\frac{(1-\rho) P}{\operatorname{tr}\left(\mathbf{D}^{1 / 2} \mathbf{Z}_{1} \mathbf{R} \mathbf{Z}_{1}^{H} \mathbf{D}^{1 / 2}\right)^{-1}}
$$

According to the results in [24, Appendix III], we obtain

$$
\operatorname{tr}\left(\mathbf{D}^{1 / 2} \mathbf{Z}_{1} \mathbf{R} \mathbf{Z}_{1}^{H} \mathbf{D}^{1 / 2}\right)^{-1} \stackrel{\text { a.s. }}{\longrightarrow} \frac{1}{\phi M} \operatorname{tr}\left(\mathbf{D}^{-1}\right)
$$

where $\phi$ is the unique solution of

$$
\phi=\frac{1}{M} \operatorname{tr}\left(\mathbf{R}\left(\mathbf{I}_{M}+\frac{K}{M} \frac{1}{\phi} \mathbf{R}\right)^{-1}\right) .
$$

Hence, the deterministic value of $\gamma^{2}$ satisfies

$$
\gamma^{2} \stackrel{a . s .}{\longrightarrow} \frac{(1-\rho) P c \phi M}{\sum_{k=1}^{K} d_{k}^{\alpha}} .
$$

Then, we evaluate the second part of the denominator in (9). Since the entries of $\tilde{\mathbf{h}}_{k}$ is independent of $\hat{\mathbf{G}}$, according to [25, Theorem 3.4], we have

$$
\begin{gathered}
\tilde{\mathbf{h}}_{k} \mathbf{R}^{1 / 2} \hat{\mathbf{G}}^{\dagger} \mathbf{s s}^{H}\left(\hat{\mathbf{G}}^{\dagger}\right)^{H}\left(\mathbf{R}^{1 / 2}\right)^{H} \tilde{\mathbf{h}}_{k}^{H} \\
\stackrel{\text { a.s. }}{\longrightarrow} \rho \operatorname{tr}\left(\mathbf{R} \hat{\mathbf{G}}^{H}\left(\hat{\mathbf{G}} \hat{\mathbf{G}}^{H}\right)^{-2} \hat{\mathbf{G}}\right) .
\end{gathered}
$$

Applying [25, Theorem 14.3], we can obtain

$$
\operatorname{tr}\left(\mathbf{R} \hat{\mathbf{G}}^{H}\left(\hat{\mathbf{G}} \hat{\mathbf{G}}^{H}\right)^{-2} \hat{\mathbf{G}}\right) \stackrel{\text { a.s. }}{\longrightarrow} \frac{1}{1-\rho} \frac{\psi}{\frac{M}{K} \phi^{2}-\psi} \frac{1}{K} \operatorname{tr}\left(\mathbf{D}^{-1}\right),
$$

where $\phi$ is given by (32) and $\psi$ is defined as

$$
\psi=\frac{1}{M} \operatorname{tr}\left(\mathbf{R}^{2}\left(\mathbf{I}_{M}+\frac{K}{M} \frac{1}{\phi} \mathbf{R}\right)^{-2}\right)
$$

Thus, for (34), we obtain

$$
\begin{gathered}
\tilde{\mathbf{h}}_{k} \mathbf{R}^{1 / 2} \hat{\mathbf{G}}^{\dagger} \mathbf{s s}^{H}\left(\hat{\mathbf{G}}^{\dagger}\right)^{H}\left(\mathbf{R}^{1 / 2}\right)^{H} \tilde{\mathbf{h}}_{k}^{H} \\
\stackrel{a . s .}{\longrightarrow} \frac{\rho}{1-\rho} \frac{\psi}{\frac{M}{K} \phi^{2}-\psi} \frac{\sum_{k=1}^{K} d_{k}^{\alpha}}{c K} .
\end{gathered}
$$

Therefore, substituting (33) and (35) into (9), we obtain (11).

\section{REFERENCES}

[1] 3GPP TR 21.101, Technical Specifications and Technical Reports for a UTRAN-based 3GPP system, Dec. 2008.

[2] 3GPP TR 36.817, Evolved Universal Terrestrial Radio Access (EUTRA); Downlink Multiple Input Multiple Output (MIMO) enhancement for LTE-Advanced, Dec. 2011.

[3] T. Yoo and A. Goldsmith, "On the optimality of multiantenna broadcast scheduling using zero-forcing beamforming," IEEE J. Sel. Areas Commun., vol. 24, no. 3, pp. 528-541, Mar. 2006.

[4] H. Gao, J. Leithon, C. Yuen, and H. A. Suraweera, "New uplink opportunistic interference alignment: An active alignment approach," in Proc. IEEE Wireless Commun. and Networking Conf. (WCNC), Shanghai, China, Apr. 2013, pp. 3099-3104.

[5] H. Gao, C. Yuen, H. Suraweera, and T. Lv, "Multiuser diversity for MIMO-Y channel: Max-min selection and diversity analysis," in Proc. IEEE Int. Conf. Commun. (ICC), Budapest, Hungary, Jun. 2013, pp. 5786-5791.

[6] H. Gao, Y. Ren, C. Yuen, and T. Lv, "A distributed user scheduling scheme for MIMO multi-way relay channel," in Proc. IEEE Int. Conf. Commun. (ICC), Sydney, Australia, Jun. 2014, pp. 4826-4831.

[7] H. Liu, H. Gao, W. Long, and T. Lv, "A novel scheme for downlink opportunistic interference alignment," in Proc. Int. Conf. Telecommun. (ICT), Lisbon, Portugal, May 2014, pp. 231-235.
[8] X. Chen, Z. Zhang, and C. Yuen, "Resource Allocation for Cost Minimization in Limited Feedback MU-MIMO Systems With Delay Guarantee," IEEE Syst. J., pp. 1-8, 2013 (early access in IEEE Xplore).

[9] K. Cheung, S. Yang, and L. Hanzo, "Spectral and energy spectral efficiency optimization of joint transmit and receive beamforming based multi-relay MIMO-OFDMA cellular networks," IEEE Trans. Wireless Commun., vol. 13, pp. 6147-6165, Nov. 2014.

[10] H. Yang and T. Marzetta, "Performance of conjugate and zero-forcing beamforming in large-scale antenna systems," IEEE J. Sel. Areas Commun., vol. 31, no. 2, pp. 172-179, Feb. 2013.

[11] F. Rusek, D. Persson, B. K. Lau, E. Larsson, T. Marzetta, O. Edfors, and F. Tufvesson, "Scaling up MIMO: Opportunities and challenges with very large arrays," IEEE Signal Processing Mag., vol. 30, no. 1, pp. 40-60, Jan. 2013.

[12] J. Zhang, C.-K. Wen, S. Jin, X. Gao, and K.-K. Wong, "On capacity of large-scale MIMO multiple access channels with distributed sets of correlated antennas," IEEE J. Sel. Areas Commun., vol. 31, no. 2, pp. 133-148, Feb. 2013.

[13] X. Su, J. Zeng, L.-P. Rong, and Y.-J. Kuang, "Investigation on Key Technologies in Large-Scale MIMO," J. Comput. Sci. Technol., vol. 28, no. 3, pp. 412-419, May 2013.

[14] O. Bai, H. Gao, T. Lv, and C. Yuen, "Low-complexity user scheduling in the downlink massive MU-MIMO system with linear precoding," in Proc. IEEE Int. Conf. Commun. China (ICCC), Shanghai, China, Oct. 2014, pp. 1-5.

[15] A. Hu, T. Lv, H. Gao, Z. Zhang, and S. Yang, "An ESPRIT-based approach for 2-D localization of incoherently distributed sources in massive MIMO systems," IEEE J. Sel. Topics Signal Process., vol. 8, no. 5, pp. 996-1011, Oct. 2014.

[16] E. Björnson, L. Sanguinetti, J. Hoydis, and M. Debbah, "Optimal design of energy-efficient multi-user MIMO systems: Is massive MIMO the answer?" Mar. 2014. [Online]. Available: http://arxiv.org/abs/1403.6150

[17] J. Nam, A. Adhikary, J.-Y. Ahn, and G. Caire, "Joint spatial division and multiplexing: Opportunistic beamforming, user grouping and simplified downlink scheduling," IEEE J. Sel. Topics Signal Process., vol. 8, no. 5, pp. 876-890, Oct. 2014.

[18] G. Lee and Y. Sung, "Asymptotically optimal simple user scheduling for massive MIMO downlink with two-stage beamforming," in Proc. Int. Workshop Signal Process. Advances in Wireless Commun. (SPAWC), Toronto, Canada, Jun. 2014, pp. 1-5.

[19] Y. Xu, G. Yue, and S. Mao, "User grouping for massive MIMO in FDD systems: New design methods and analysis," IEEE Access, vol. 2, pp. 947-959, 2014.

[20] M. Kobayashi, N. Jindal, and G. Caire, "Training and Feedback Optimization for Multiuser MIMO Downlink," IEEE Trans. Commun., vol. 59, pp. 2228-2240, Aug. 2011.

[21] T. Marzetta, "How much training is required for multiuser mimo?" in Proc. Asilomar Conf. Signals, Systems and Computers (ACSSC), Pacific Grove, CA, Oct. 2006, pp. 359-363.

[22] H. Huh, "Large system analysis of multi-cell MIMO downlink: fairness scheduling and inter-cell cooperation," PhD dissertation, University of Southern California, 2012.

[23] A. V. Zelst and J. S. Hammerschmidt, "A single coefficient spatial correlation model for multiple-input multiple-output (MIMO) radio channels," in Proc. URSI XXVIIth General Assembly, 2002, pp. 1-4.

[24] S. Wanger, R. Couillet, M. Debbah, and D. Slock, "Large system analysis of linear precoding in correlated MISO broadcast channels under limited feedback," IEEE Trans. Inf. Theory, vol. 58, pp. 45094537, Jul. 2012.

[25] R. Couillet and M. Debbah, Random Matrix Methods for Wireless Communications, 1st ed. Cambridge, United Kingdom: Cambridge University Press, 2011.

[26] M. Biguesh and A. Gershman, "Training-based MIMO channel estimation: a study of estimator tradeoffs and optimal training signals," IEEE Trans. Signal Process., vol. 54, pp. 884-893, Mar. 2006.

[27] K. B. Baltzis, "Hexagonal vs circular cell shape: a comparative analysis and evaluation of the two popular modeling approximations," in Cellular Networks-Positioning, Performance Analysis, Reliability. Rijeka, Croatia: InTech, 2011, ch. 4, pp. 103-122.

[28] L. Zhao, K. Zheng, H. Long, and H. Zhao, "Performance analysis for downlink massive MIMO system with ZF precoding," Trans. Emerging Telecommun. Tech., vol. 25, pp. 1219-1230, Dec. 2014.

[29] M. Jung, Y. Kim, J. Lee, and S. Choi, "Optimal number of users in zeroforcing based multiuser MIMO systems with large number of antennas," J. Commun. and Networks, vol. 15, no. 4, pp. 362-369, Apr. 2013.

[30] "Gauss hypergeometric function." [Online]. Available: http://functions. wolfram.com/HypergeometricFunctions/Hypergeometric2F1/ 
[31] O. Sjobergh, E. Jorswieck, and E. Larsson, "Greedy user selection for zero-forcing and MMSE multiuser beamforming with channel estimation errors," in Proc. IEEE Int. Conf. Acoustics, Speech and Signal Process. (ICASSP), Las Vegas, Nevada, Mar. 2008, pp. 3137-3140.

[32] A. Tomasoni, G. Caire, M. Ferrari, and S. Bellini, "On the selection of semi-orthogonal users for zero-forcing beamforming," in Proc. IEEE Int. Symp. Inf. Theory (ISIT), Seoul, Korea, Jun. 2009, pp. 1100-1104.

[33] 3GPP TR 36.814, Further advancements for E-UTRA physical layer aspects (Release 9), Mar. 2010.

[34] R. Jain, D. Chiu, and W. Hawe, "A quantitative measure of fairness and discrimination for resource allocation in shared computer systems," DEC, Tech. Rep. TR-301, Sep. 1984.

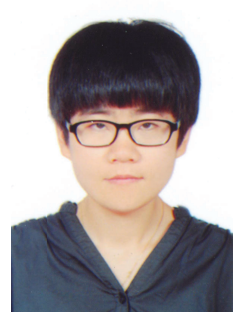

Haijing Liu received her B. S. and M.S. degrees in Communication and Information System from Southeast University, Nanjing, China, in 2007 and 2010, respectively. From 2010 to 2012, she worked in Alcatel-Lucent Shanghai Bell Co. Ltd as an hardware engineer. She is currently pursuing the $\mathrm{Ph} . \mathrm{D}$. degree at Beijing University of Posts and Telecommunications, Beijing, China. Her research interests include massive MIMO systems and lowcomplexity digital signal processing in wireless communications.

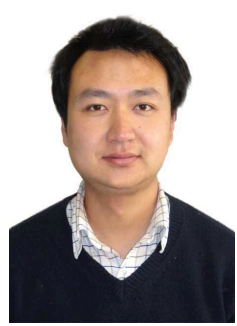

Hui Gao (S'10-M'13) received the B. Eng. degree in information engineering and the Ph.D. degree in signal and information processing from Beijing University of Posts and Telecommunications (BUPT), Beijing, China, in July 2007 and July 2012, respectively. From May 2009 to June 2012, he also served as a Research Assistant for the Wireless and Mobile Communications Technology R\&D Center, Tsinghua University, Beijing, China. From April 2012 to June 2012, he visited Singapore University of Technology and Design (SUTD), Singapore, as a Research Assistant. From July 2012 to February 2014, he was a Postdoc Researcher with SUTD. He is now with the School of Information and Communication Engineering, BUPT, as an Assistant Professor. His research interests include massive MIMO systems, cooperative communications, ultrawideband wireless communications.

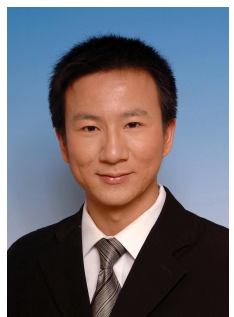

Shaoshi Yang (S'09-M'13) received the B.Eng. Degree in Information Engineering from Beijing University of Posts and Telecommunications (BUPT), China, in 2006, the first Ph.D. Degree in Electronics and Electrical Engineering from University of Southampton, U.K., in 2013, and a second Ph.D. Degree in Signal and Information Processing from BUPT in 2014. Since 2013 he has been a Postdoctoral Research Fellow in University of Southampton, U.K., and from 2008 to 2009, he was an Intern Research Fellow with the Intel Labs China, Beijing, where he focused on Channel Quality Indicator Channel design for mobile WiMAX $(802.16 \mathrm{~m})$. His research interests include MIMO signal processing, green radio, heterogeneous networks, cross-layer interference management, convex optimization and its applications. He has published in excess of 30 research papers on IEEE journals and conferences.

Shaoshi has received a number of academic and research awards, including the PMC-Sierra Telecommunications Technology Scholarship at BUPT, the Electronics and Computer Science (ECS) Scholarship of University of Southampton and the Best $\mathrm{PhD}$ Thesis Award of BUPT. He serves as a TPC member of a number of IEEE conferences and journals, including IEEE ICC, PIMRC, ICCVE, HPCC and IEEE Journal on Selected Areas in Communications. He is also a Junior Member of the Isaac Newton Institute for Mathematical Sciences, Cambridge University, UK. (https:// sites.google.com/site/shaoshiyang/)

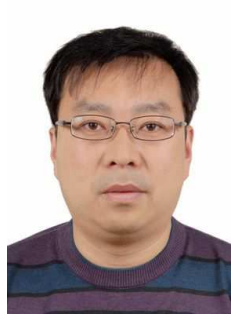

Tiejun Lv (M'08-SM'12) received the M.S. and $\mathrm{Ph} . \mathrm{D}$. degrees in electronic engineering from the University of Electronic Science and Technology of China (UESTC), Chengdu, China, in 1997 and 2000, respectively. From January 2001 to December 2002, he was a Postdoctoral Fellow with Tsinghua University, Beijing, China. From September 2008 to March 2009, he was a Visiting Professor with the Department of Electrical Engineering, Stanford University, Stanford, CA, USA. He is currently a Full Professor with the School of Information and Communication Engineering, Beijing University of Posts and Telecommunications (BUPT). He is the author of more than 200 published technical papers on the physical layer of wireless mobile communications. His current research interests include signal processing, communications theory and networking. He was the recipient of the Program for New Century Excellent Talents in University Award from the Ministry of Education, China, in 2006. 